\title{
Battery Lifecycle Framework: A Flexible Repository and Visualization Tool for Battery Data from Materials Development to Field Implementation
}

\author{
Valerio De Angelis ${ }^{1}$, Yuliya Preger ${ }^{1}$, and Babu R. Chalamala ${ }^{1}$ \\ ${ }^{1}$ Energy Storage Technology and Systems, Sandia National Laboratories, Albuquerque, New \\ Mexico, United States of America \\ Correspondence: vdeange@sandia.gov
}

\begin{abstract}
The Battery Lifecycle (BLC) Framework is an open-source platform that provides tools to visualize, analyze, and share battery data through the technology development cycle, including data from material characterization, cell testing, manufacturing, and field testing. The BLC framework provides users with a unified view of their data that they can use to optimize materials and cell configurations, validate cell performance under application conditions, and mitigate manufacturing variations and field failures. BLC has four components: data importers, one or more databases, a front-end for querying the data and creating visualizations, and an application programming interface to process the data. Instead of building the system from the ground up, we developed BLC around Redash, a robust open-source extract-transform-load engine. BLC has been deployed for two applications: (i) tracking the development of a single battery technology from the lab to a manufacturing line and systems installed in the field, and (ii) comparing studies of multiple cells of the same battery chemistry and configuration. The latter implementation is publicly available at www.BatteryArchive.org. The open-source software package is available at: https://github.com/battery-lcf/battery-archive-sandbox.
\end{abstract}

\section{Motivation and Significance}

Rechargeable batteries are central to modern society, powering consumer electronics, and increasingly, electric vehicles and stationary energy storage applications. The growing demand for rechargeable batteries has inspired the development of new battery technologies that show significant promise in laboratory prototypes, but struggle to scale-up and reach the performance needed for commercial products. In fact, the process of scaling up and integrating emerging technologies from lab prototypes into commercial products has traditionally taken a decade or longer. The Energy Storage Grand Challenge Roadmap, released by the U.S. Department of Energy in 2020, challenged the energy storage industry to accelerate battery development by providing better integration of activities from material development (lab cells) to device testing (batteries), system deployment, and market rollout. ${ }^{1}$ Unfortunately, the information collected at each step of the development cycle is not easily sharable.

Fig. 1a lays out four key steps of the battery development process. Technology progresses from electrodes, to lab cells, to manufacturing, and to systems. Data collected at every step can inform the development of other steps. For example, data from systems in the field can be used to quantify the cell-to-cell variation and cell failure and optimize manufacturing processes. System 
data can also be used to develop accelerated protocols to validate cell performances before they are mass produced. Finally, the comparison of material characterization and cell testing data can be used to optimize materials and cell fabrication before large investments in manufacturing plants are made. However, these feedback loops cannot occur if the data is not all accessible in one place. Several useful battery analysis tools that leverage open source software have been published in the last few years. For example, PyBaMM, developed by the Faraday Institution, provides open-source tools to compare Li-ion and Lead-acid battery models with experimental data. ${ }^{2}$ CellPy is an open source Python Package developed by researchers at the Institute for Energy Technology in Norway to interpret and handle data from cycling tests of batteries and cells. ${ }^{3}$ BEEP is an open-source package from the Toyota Research Institute designed to prepare battery data and metadata for machine learning packages. ${ }^{4}$ Both CellPy and BEEP offer tools to pre-process battery data from battery testers for scientific use. These tools validate the integrity of the data, but do not visualize the data. Other notable efforts include: Galvanalyser, developed by the Battery Intelligence Lab at the University of Oxford, which proposes a browsable version of a battery database for laboratory use and designed around their specific database schema ${ }^{5}$, and the Universal Battery Database developed by the Dahn Group at Dalhousie University. ${ }^{6}$ The latter two projects target small user groups and provide support for pre-determined plots, but lack the ability to easily add new visualizations to compare data across institutions and applications. These tools have greatly helped battery research groups with functions including analysis of experimental battery test results and model validation. However, none of these tools are flexible enough to provide a holistic view of the data generated during the various stages of development (Fig. 1a).

In this paper, we lay out a Battery Lifecycle Framework (BLC) that can be used to track battery data through their full lifecycle from materials to systems. BLC is currently implemented in two configurations. In one implementation, BLC was used by a battery group to track data from the lab to the field. Users imported data from battery testers and data from the manufacturing process into a single dashboard. They correlated quality control test results with cycle data results and manufacturing process data. In a second implementation, the framework was used to build and publish an online repository of cell testing data at Battery Archive. www.BatteryArchive.org was launched in September 2020 and exceeded 1,000 users within a few months. The site was created as a public repository for easy visualization, analysis, and comparison of battery degradation data across institutions. Several groups contacted us to learn how to replicate a system like Battery Archive for private use. Motivated by these inquires, we are making the framework widely available to the community. In Section 2, we discuss the framework architecture. In Sections 3 and 4, we describe two distinct implementations of BLC.

\section{Battery Lifecycle Framework}

We decided to base BLC on open-source packages developed by the large software community instead of developing tools from the ground up. Initially, we considered data science web tools like D3.js ${ }^{7}$ and plotly ${ }^{8}$ but realized that we needed to develop a suite of back-end tools to connect data sources and build structured queries. We also evaluated cube.js, ${ }^{9}$ a powerful tool to add user-facing analytics to existing applications, but quickly realized that it lacked robust rendering and a user interface that could be used to build new views on the data. We finally settled on 
Redash, which combines elements of plotly and cube.js with a robust management web interface. Redash is an open-source extract-transform-load (ETL) tool that can be used to manage data connections and generate data visualizations. ${ }^{10}$ Redash offered the foundation around which we built battery-specific software tools.

The overall architecture of the BLC is shown in Fig. 1b. Data are stored in one or more data sources that are connected to Redash and a Python Application Programming Interface (API) accessible from Jupyter Notebook. The Redash web interface contains web tools designed to add data connections, query the data, use the results of the queries in plots, and publish the plots in public and private dashboards. The data shown in Figures 2, 4, and 6 have all been generated using Redash web interface tools summarized in Fig. 1b.

(a)

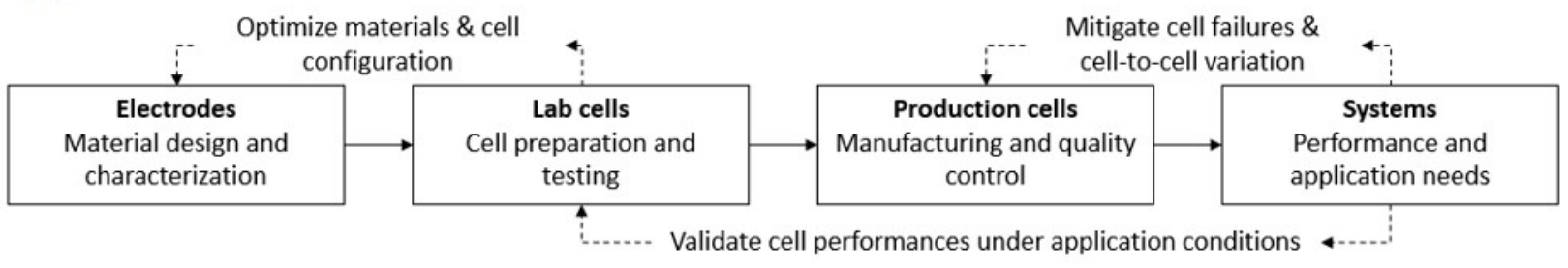

(b)

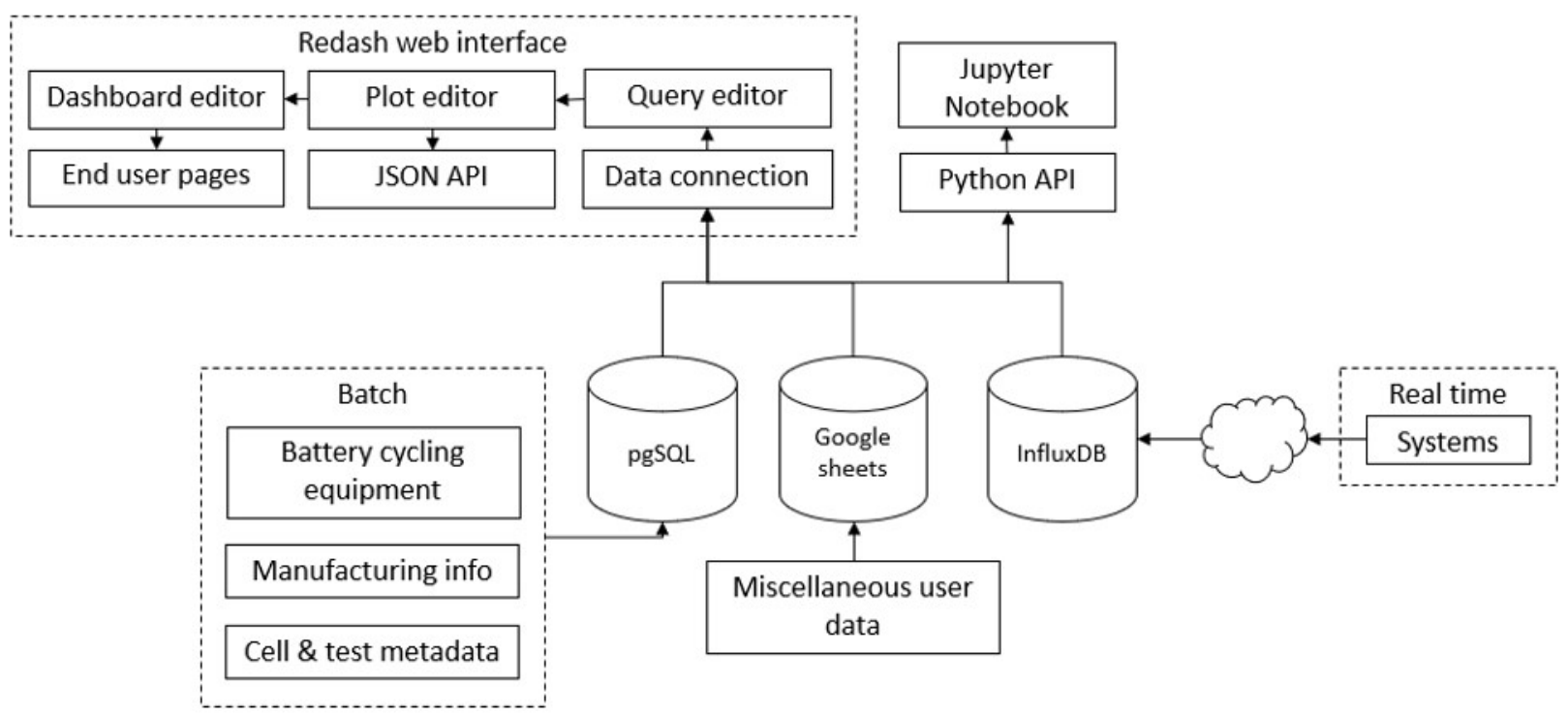

Fig. 1. (a) Battery development lifecycle. Dashed lines represent feedback loops intended to shorten the development cycle; (b) Architecture of the Battery Lifecycle Framework for linking battery data and metadata from the lab to the field.

We have used three types of data sources so far. BLC accepts batch data that is stored in a Postgres database (pgSQL), real-time data that is stored in InfluxDB, and miscellaneous user data that is stored in Google Sheets. pgSQL is used to store relational data, such as data from battery testers or manufacturing lines, including information that characterizes the cell and the test (metadata). pgSQL was selected for its ability to add extensions and to mix relational (tabulated content) and non-relational data (name-value pairs like compositions or cell preparation 
details). So far, we have developed batch importers for the following formats: Excel (including Arbin and MACCOR battery tester outputs), CSV, and MATLAB files, and Oracle and Access databases. InfluxDB is used to store timeseries data from systems in the field, given its ability to hold large quantities of JSON (name-value pair) data. Data are imported in InfluxDB by installing telegraf on remote systems. Miscellaneous user data are stored in Google Sheets. Google Sheets are an easy way to enter data like model results or additional cell metadata that can then be associated to the data in pgSQL or InfluxDB. In other words, Google Sheets are used like a data scrapbook for BLC to give users flexibility to enter data specific to their needs. The data stored in any of the data sources can be combined and rendered in graphs and tables on web dashboards using the Redash web interface, and also made available to other web systems using the Redash JSON API. ${ }^{11}$ Finally, analysis tools can access the data in Jupyter Notebook through a Python API. In the following subsections, we discuss some of the key implementation components of BLC.

\subsection{Design of pgSQL}

As discussed previously, we selected Postgres to store relational data. A current implementation of the database includes the following tables: cell_metadata for Cell Information, test_metatada for Test Information; cycle_data for cycle number, energy, capacity, max/min voltage, and current; timeseries_data for test and cycle time, current, voltage, and charge/discharge capacity and energy; manufacturing_data for information from a manufacturing line; and system_data for information about systems in the field. The current schema is on the associated GitHub.

All the tables use Cell ID as the common tag. The Cell ID is a VARCHAR (string) field that offers some basic description of the cell. For example, Battery Archive uses the format \{Source\}_\{Form Factor\}_\{Composition\}_\{Test conditions\}_\{Duplicate index $\}$. In another implementation, the format was $\{$ Researcher_initials\}_\{Anode \}_\{Cathode $\} \_\{\text {Electrolyte\}. Using a string as the Cell ID }$ makes it easy to interpret data, preserve the origin of the cell, and make data more readable. The database uses the Cell ID as an index on all the tables. Using an index on the tables is necessary (especially for the timeseries_data and cycle_data tables) to improve the performance of the data SELECT queries, as, in most cases, data are queried by Cell ID. An additional numeric index could have been created instead of using the Cell ID to relate and index cells in pgSQL. However, the use of an additional field as an index would have made SQL queries more complex and prevented users with moderate knowledge of SQL from creating new queries specific to their needs.

The simplicity of the queries comes at the expense of performance. Indices on text fields are more expensive than indices on numeric fields and can make UPDATE queries slow. This problem is typically encountered when the number of cells increases past a few hundred. During an update, pgSQL generates new records, rebuilds the index, and keeps a transaction log in case rollback is needed. After these steps are completed, Postgres removes the old data. ${ }^{12}$ The process is time intensive when large amounts of data are updated. Therefore, special care needs to be taken to update records in the time series data when needed (a rare occurrence) with the schema used by BLC. We found that the optimal solution to update time series data is to copy the existing records in a Data Frame and swap table (to recover the data if an error takes place), update the 
data in the Data Frame, then finally delete the old data and add the new data in Postgres. The database schema and the code used to update records in the database are available on GitHub. We included a sample populated database to help groups get started before they import their own data.

\subsection{Batch import of data}

The relational data stored in the BLC pgSQL database can come from several sources. In Section 4 , we show data imported from a FileMaker database and how it can be combined with data imported from battery cyclers. Different groups may have the need to build unique importers and there are many edge cases, so the importer available in the GitHub repository (for common battery cycling equipment) is only intended to serve as a model.

Battery cycling equipment is designed to test batteries under prescribed protocols where charge and discharge cycles alternate. All battery testers store basic information (time stamp, test time, voltage, current, test and cell temperature, and cycle index). The Python script in the BLC Github is used to import Excel files generated by Arbin testers. The importer can process multiple cells and multiple Excel files per cell. The cells to be processed are listed in the cell_list Excel document that contains metadata and test file location. For each cell, the test files are loaded in Python Dataframe structures, merged, and sorted by date time. Then the cycle index and test time is rebuilt. Finally, as sometimes the files contain multiple rows with the same time stamp, data are resorted by the rebuilt test time. After the data is validated and the statistics are calculated, the data are stored in the cell_metadata, test_metadata, cycle_data, and timeseries_data tables.

\subsection{Design of data queries in Redash}

Data from the data connections in Fig. 1b can be accessed directly from the Redash Query Design page. The editor accepts standard SQL queries. We built queries optimized to generate quantities used by the battery industry. The queries are in GitHub and also loaded in the BLC docker. These queries can serve as a model to users of BLC who need to query other quantities or data sources. For example, Fig. 2a contains the SQL Query that gathers the data for cycle voltage and current. When a parameter is added to the query, a corresponding dropdown is automatically associated with the query. The query in Fig. $2 a$ uses four parameters $\left\{\left\{c e l l \_i d\right\}\right\},\{\{\min \}\},\{\{\max \}\}$, and $\{\{$ sample_vc $\}$. 
(a)

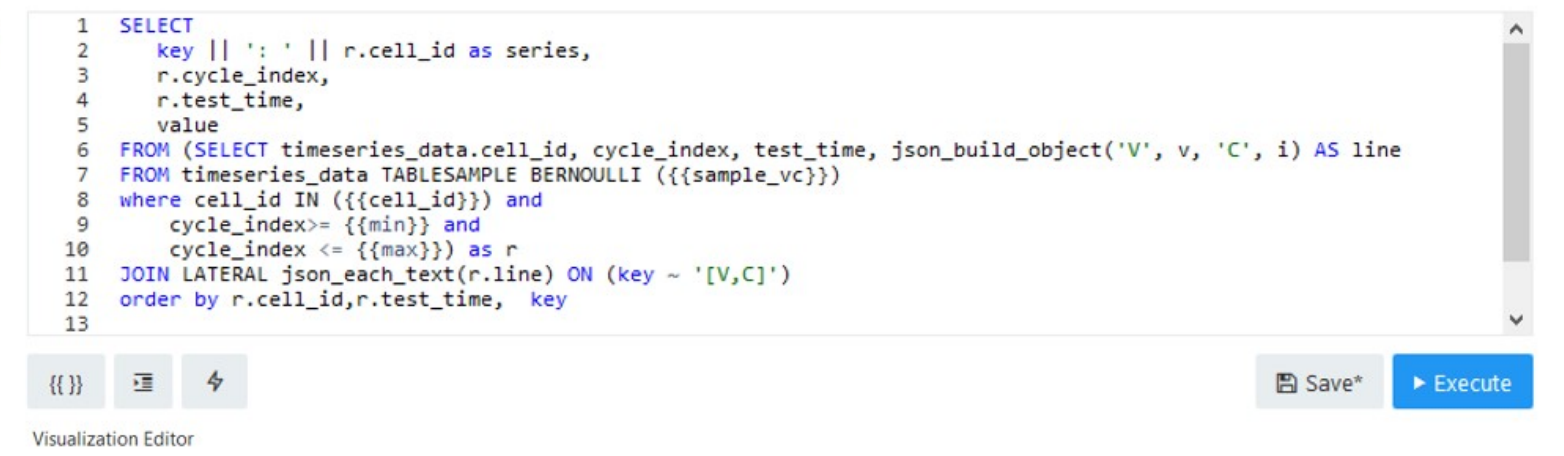

(b)

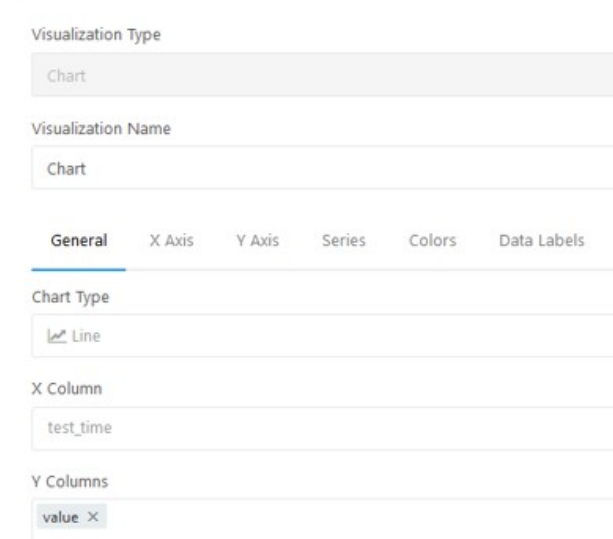

(c)

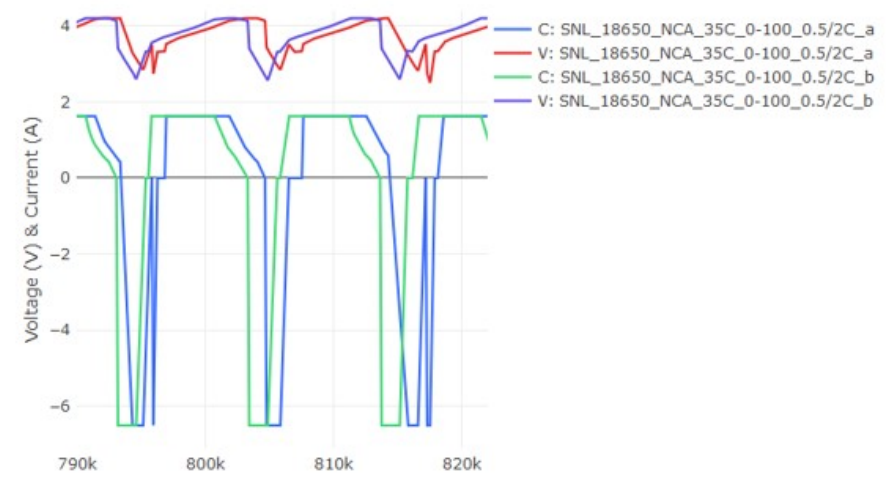

Fig. 2. (a) Example query to extract voltage and current data from the cell database. The Redash web interface allows parameter-based queries. In this case, Cell id, min (start) and max (end) cycle, and data sample \% are parameters; (b) Interface to create graphs using the results of a query; (c) Example plot of voltage versus cycle time. The plots can be combined in dashboards like those in Figures 4 and 6.

The site uses two built-in features of pgSQL to optimize the queries: the ability to combine JSON and relational data, and the ability to down sample data. To plot any number of quantities for any number of cells, json_build_object groups data for a cell in a JSON object and JOIN Lateral splits the data on multiple rows. ${ }^{13}$ As a result, the query returns records in the format cell quantity label: cell_id, cycle time stamp, test time stamp, and value that can then be easily plotted regardless of the number of cells or quantities, as shown in Fig. 2c. The built-in down sampling features of pgSQL control the number of datapoints returned to the browser. In the current implementation of BLC, the TABLESAMPLE BERNULLI option returns a random subset of data selected from the entire result space. ${ }^{14}$ In a future iteration, the custom extension feature of pgSQL will be used for down sampling based on the voltage-current curve gradients to return a constant number of points regardless of the time period selected. The source code for all the queries used to generate the graphs in this manuscript is available on GitHub. Finally, the interfaces in Fig. $2 b$ and $2 c$ demonstrate how easy it is for non-computer experts to build custom queries and graphs in Redash. Redash also includes a dashboard editor that can be used to combine the graphs and tables generated from the query editor in public (Fig. 4) or private (Fig. 6) dashboards. 


\subsection{Versatile use of Google Sheets}

Users can connect Google Sheets to Redash. The data from the Google Worksheet can be queried using the format google_sheet_id|sheet_number after the Google API JSON Key file is added as a data connection in the Redash 'add connection' interface. This is a versatile option that we added to BLC so users can add miscellaneous data and compare it with data in other data sources. For example, Fig. 3 shows a comparison between experimental data in pgSQL and model results extracted directly from a Google Worksheet.

Tanks-in-Series battery model

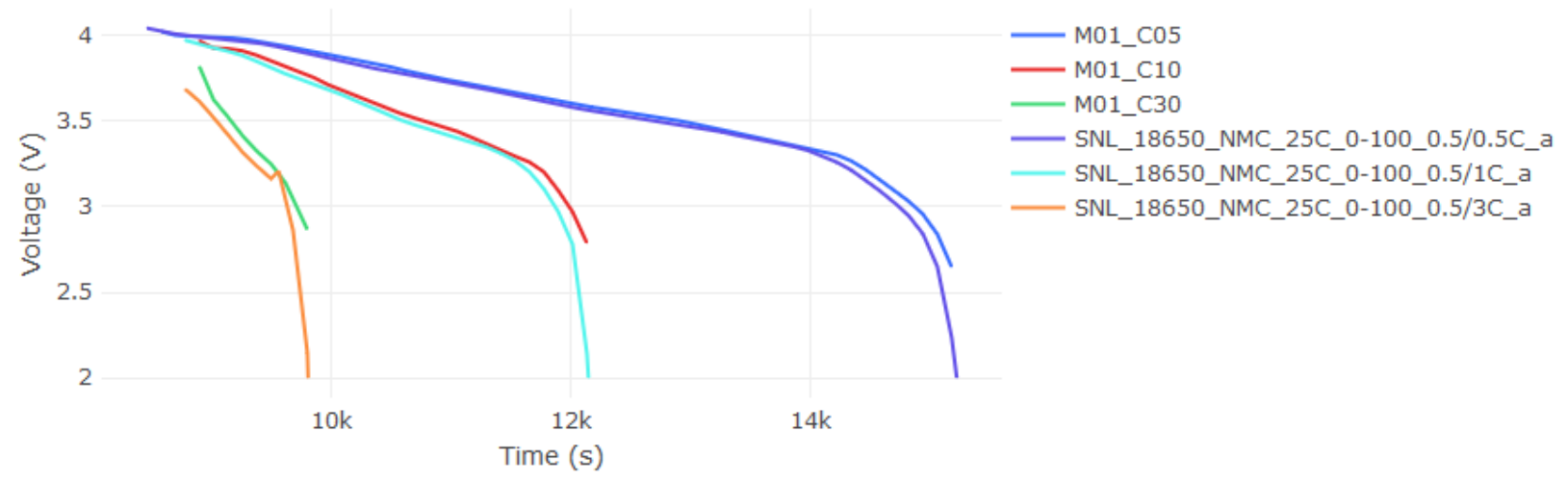

¿just now

Fig. 3. Query of data from different sources, in this case, experimental data and models. The model data is read directly from a Google Sheet and the experimental data is from pgSQL.

In another example, a group used Google Sheets to maintain a list of cells to be displayed in a specific Redash dashboard. The Cell ID entered in the Google Sheet database can be used in SQL queries like the one in Fig. 2a to filter the data to the cells in the Google Sheet and create dashboards designed for specific studies. This is a simple method to create dashboards that follow the evolution of different studies or to add additional metadata.

\subsection{JSON API}


The queries generated in the front-end query design tool (Fig. 2) can also be called via the Redash JSON API. For example, the code in Snippet 1 is used to return the results from a query and accept the parameters used in the query (for example Cell_id). The feature allows data stored in BLC to be used in other systems. For example, data returned from a Redash query can be used directly in Google Sheets.

def get_fresh_query_result(redash_url, query_id, api_key, params):

$\mathrm{s}=$ requests.Session()

s.headers.update(\{'Authorization': 'Key \{\}'.format(api_key)\})

response = s.post('\{\}/api/queries/\{\}/refresh'.format(redash_url, query_id), params=params)

print(response.url)

print(response.text)

if response.status_code $!=200$ :

raise Exception('Refresh failed.')

result_id $=$ poll_job(s, redash_url, response.json()['job'])

if result_id:

response $=$ s.get('\{\}/api/queries $/\{\} /$ results/ \{\} .json'.format(redash_url, query_id, result_id))

if response.status_code $!=200$ :

raise Exception('Failed getting results.')

else:

raise Exception('Query execution failed.')

return response.json()['query_result']['data']['rows']

\section{Example implementation: Battery Archive public site}

www.BatteryArchive.org uses the BLC framework to provide a public repository for easy visualization, analysis, and comparison of battery degradation data across institutions. The Battery Archive site was released in September 2020 and currently includes battery cycling data from the Center for Advanced Life Cycle Engineering (CALCE) ${ }^{15,16}$, the Hawaii Natural Energy Institute $(\mathrm{HNEI})^{17}$, Oxford University $(\mathrm{OX})^{18,19}$, and Sandia National Laboratories (SNL). ${ }^{20}$ We are in the process of uploading data from Purdue University ${ }^{21}$. University College London (UCL) ${ }^{22}$, UC Berkeley (CAL) ${ }^{23}$, and NASA. ${ }^{24}$ Various other datasets are in the pipeline. The data is stored in Postgres using the schema in Section 2.1 and the importers in Section 2.2.

The data returned from the queries is organized in two public dashboards. One dashboard contains a list of Cell IDs with cell and test metadata. The cells can be filtered by the metadata. Users can click on a cell and access the cell data in a second dashboard. The cell data dashboard contains cycle data and time series quantities that are commonly used by the battery community, including energy and capacity efficiency, and a comparison of voltage curves over time. 
Screenshots from the dashboards are in Fig. 4. All the graphs and tables were generated using the tools in Fig. 2.

(a)

\section{Cell List}

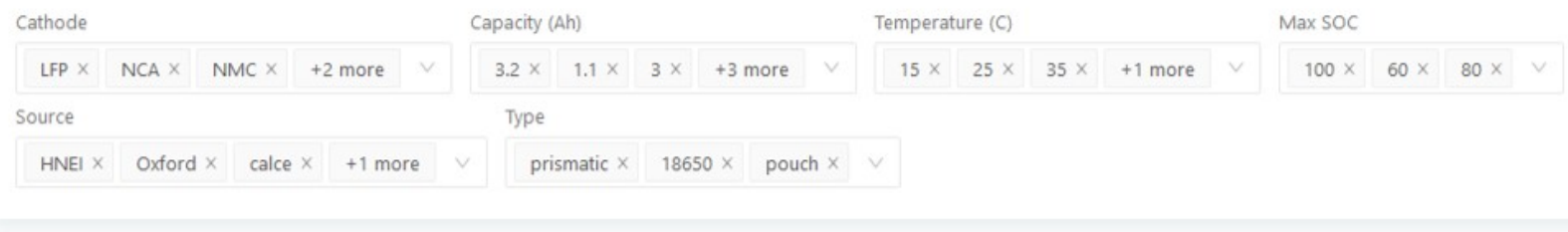

Li-ion cell list

$\begin{array}{lrll}\text { Cell ID } & \text { Cycles } & \text { Cathode } & \text { Anode } \\ \text { HNEL_18650_NMC_LCO_25C_0-100_0.5/1.5C_a } & 1,113 & \text { NMC-LCO } & \text { graphite } \\ \text { HNEL_18650_NMC_LCO_25C_0-100_0.5/1.5C_b } & 1,108 & \text { NMC-LCO } & \text { graphite }\end{array}$

(b)

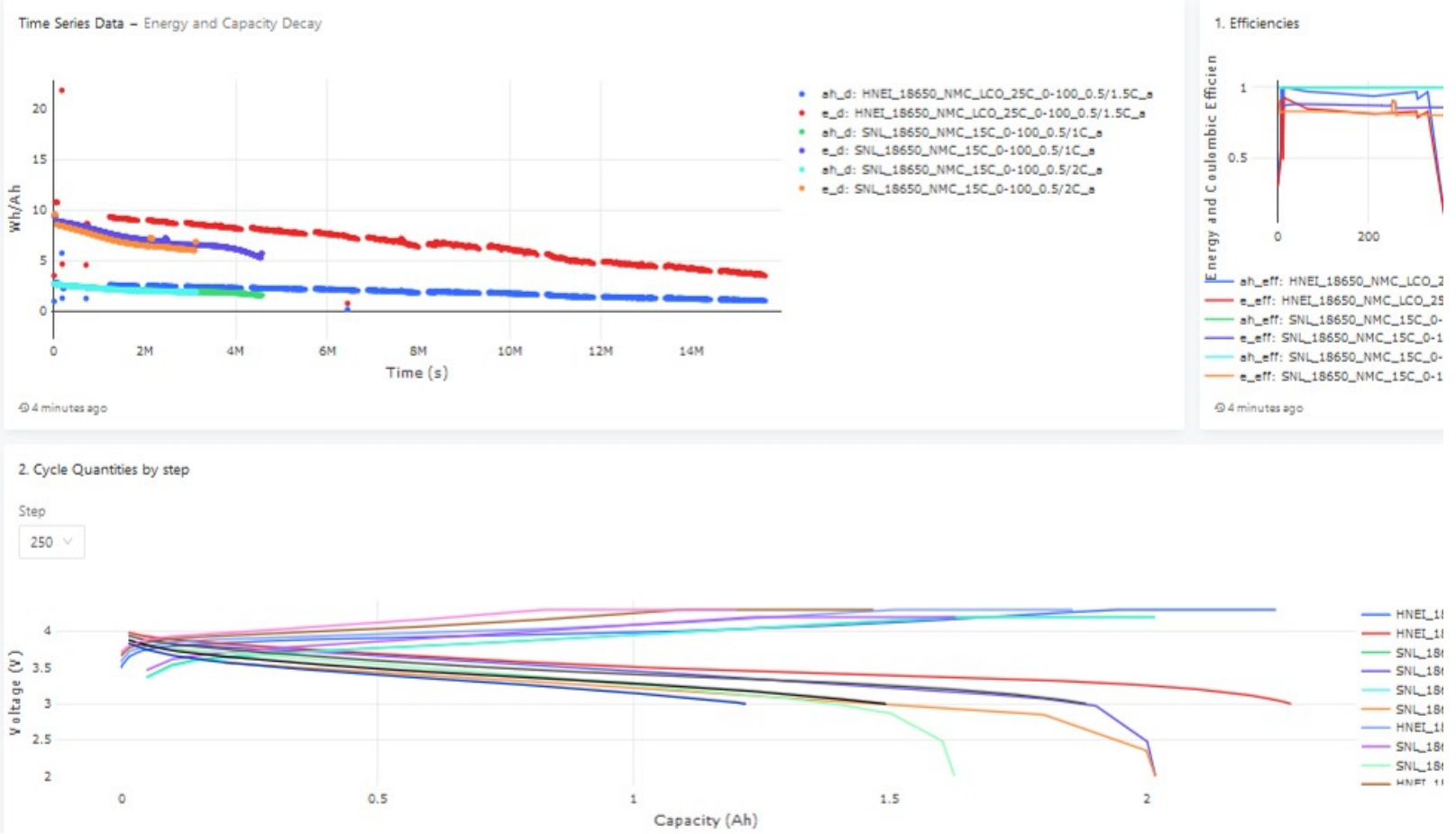

Fig. 4. Dashboards in the Battery Archive public site showing (a) a list of cells with metadata for filtering and (b) plots of cell data including time series and cycle-specific quantities.

\subsection{Use of the Python API}

The data in Battery Archive can be used to illustrate the value of the Python API for accessing data from Jupyter Notebook. For example, we used a Jupyter Notebook session on Battery Archive to compare the capacity fade of two datasets, one from HNEI and one from SNL. The cycling protocols utilized by the studies include a periodic capacity-check cycle. However, the two 
studies used different definitions of the capacity-check cycle. The SNL study used a chargedischarge sequence, and the HNEI study had a partial discharge followed by a charge-discharge sequence, as illustrated in Fig. 5 a.

(a)

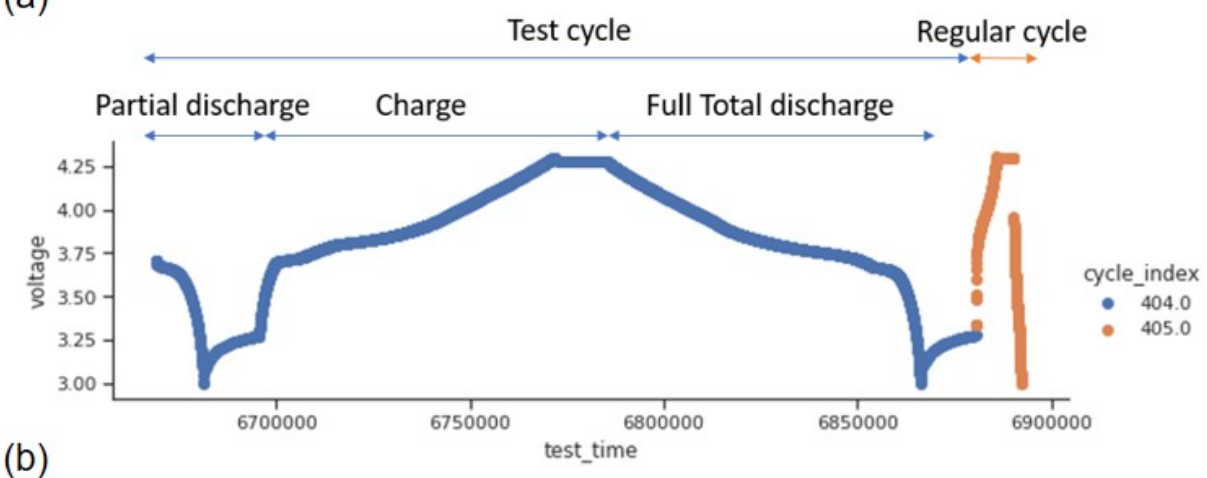

(b)

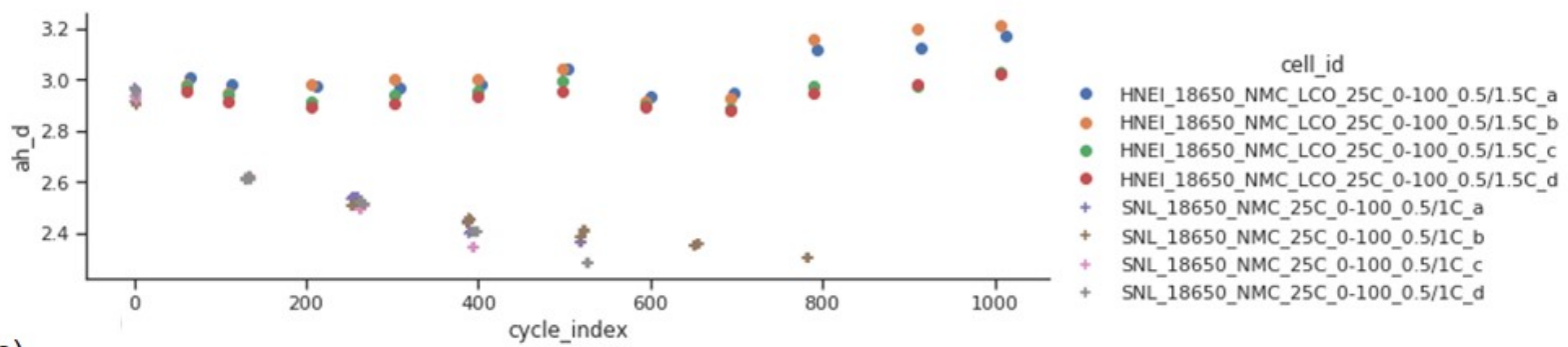

(c)

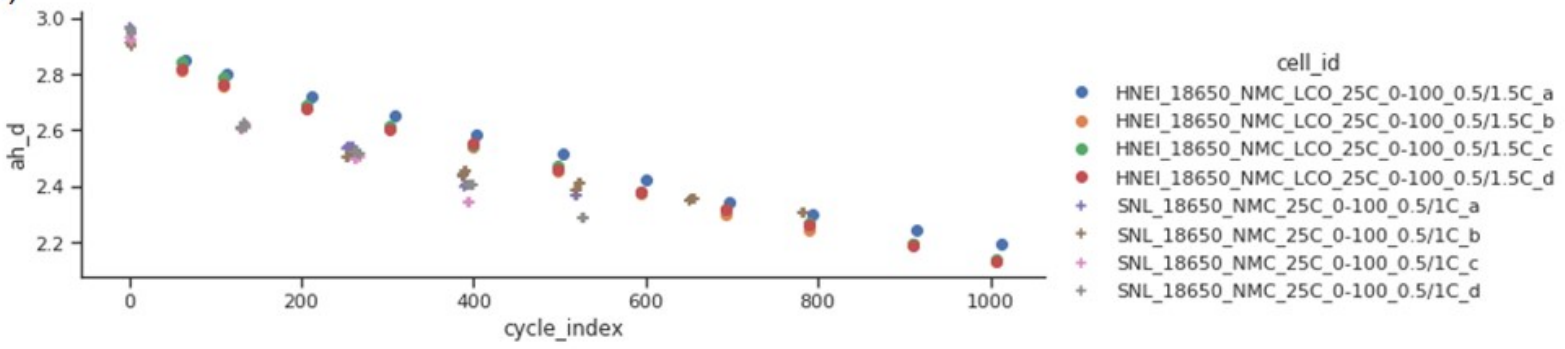

Fig. 5. (a) Time series data for a cell from the HNEl study. The capacity-check cycle includes an initial partial discharge that needs to be filtered before data can be compared with other studies; (b) Discharge capacity comparison for the HNEI and SNL cells. The capacity (ah_d) is calculated using the total discharge capacity in the capacity-test cycle. The HNEl study's capacity is overestimated as it includes the contribution of the initial partial discharge; (c) Discharge capacity comparison for the HNEI and SNL cells. The capacity (ah_d) is calculated using the total discharge capacity in the capacity-test cycle for the SNL cells and the first full discharge capacity for the HNEI cells.

A direct comparison of the two studies' discharge capacity will lead to the results shown in Fig. $5 \mathrm{~b}$, where the HNEI cells' capacities are overestimated. However, after the data are loaded in Jupyter Notebook, the first partial discharge can be filtered and the correct discharge capacity calculated. The final comparison of capacity fade (after the initial partial discharge in the HNEI cells is filtered out) is shown in Fig. 5c. The Jupyter Notebook file to complete the calculations in Fig. 5 using the sample data in the reduced database is in the GitHub. 


\section{Example implementation: tracking multiple data streams}

In this section we describe how BLC can be used to track a single chemistry through development, manufacturing, and systems. As shown in Fig. 6, manufacturing data, cell metadata, cycle data, and system data can all be displayed in one dashboard. Fig. 6a shows the manufacturing data imported into pgSQL from a FileMaker database. Fig. 6b shows the cell testing data imported from Arbin testers (MS Access) and a PEC tester (Oracle Database). Data from systems are stored in InfluxDB and shown in Figure 6c.

(a)

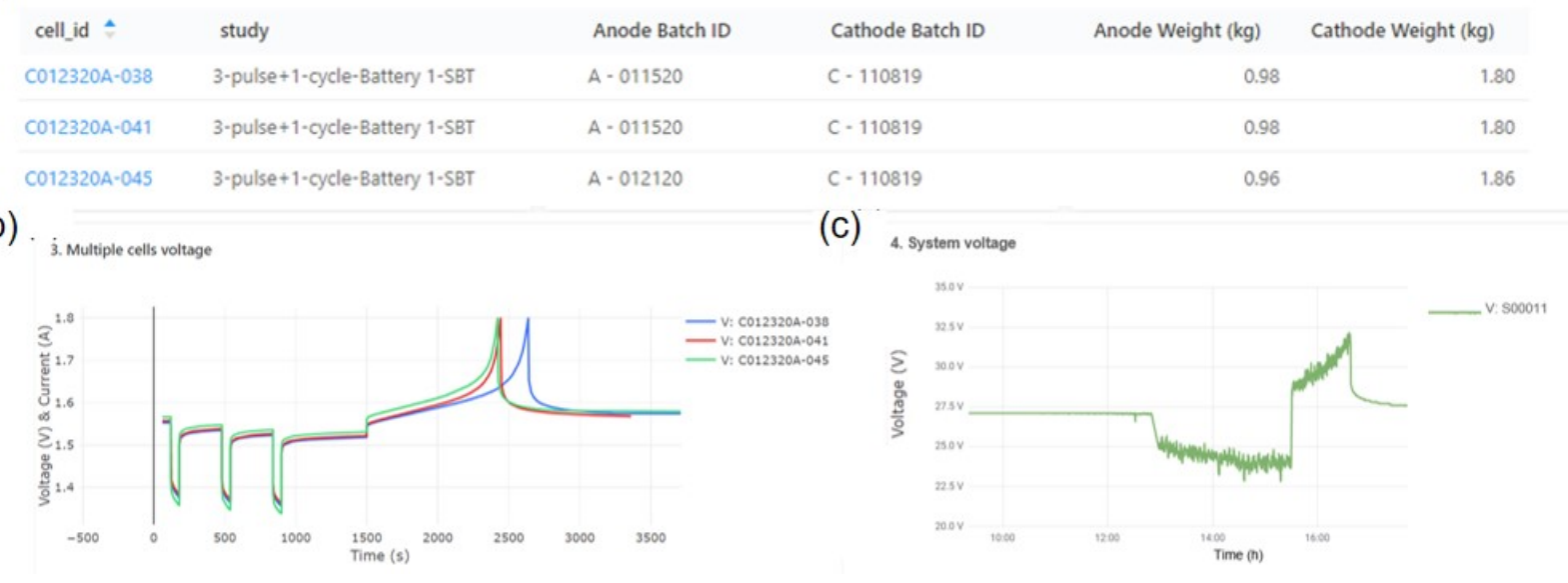

Fig. 6. (a) Manufacturing data for 3 cells; (b) Pulse QC test for the same 3 cells imported from PEC and Arbin testers; (c) Data from the fielded system that uses the 3 cells in (a) and (b).

In this case, there is no clear correlation between anode and cathode weights (Fig. 6a) and the 3-pulse test (Fig. 6b). The ability to access the data in Jupyter Notebook can lead to more insights as the entire dataset can be analyzed. The data collected during the QC tests and the manufacturing metadata can then be correlated with system information.

\section{Conclusion}

In this work, we introduced the Battery Lifecycle Framework, an open source project that provides tools to link battery data and metadata across the various stages of battery development, from laboratory prototypes to fielded systems. The framework leverages existing open-source tools like Redash that we augmented with components optimized to import, display, and analyze battery data. We discussed two implementations of this framework: tracking a single technology during its development and comparing battery data from different studies in the Battery Archive public site. We are releasing the tools to build a site like Battery Archive as an open source project. We envision that multiple groups will run the framework on their servers and share data sources and dashboards as appropriate while keeping some of their data private. We are currently collaborating with the battery R\&D and manufacturing community to define a common metadata schema that will ensure data standardization and interoperability with other open source battery analysis tools. 


\section{Software Availability}

The open-source software package is available at: https://github.com/battery-lcf/battery-archivesandbox. The code and documentation in the repository can be used to build and operate a site like BatteryArchive.org. The installer will create and start docker containers with Redash (the web interface) and a postgres database with a basic schema designed for battery data. It also contains a script to add data from Excel files generated by Arbin testers. The repository includes a stepby-step installation guide. This starter package is intended to familiarize users with various aspects of Redash for batteries so that they may design their own application-specific dashboards and queries, such as those in Figure 6.

\section{Acknowledgments}

This work was supported by the U.S. Department of Energy, Office of Electricity, Energy Storage Program under the direction of Dr. Imre Gyuk. BLC development started when Dr. De Angelis was with CUNY Energy Institute at the City College of New York. He would like to thank Prof. Sanjoy Banerjee for his encouragement to pursue the work and Dr. Jinchao Huang, Andreas Savva, and Dr. Gautam Yadav for testing an early version of the framework. We thank Irving Derin for his help with the BLC docker container. We thank Sam Roberts-Baca (Sandia) for help with the development of the front-end pages of the Battery Archive site and Mark Spoonamore (Sandia) for advice on database structure. We thank Dr. Matthieu Dubarry of the Hawaii Natural Energy Institute for the data in Fig. 5 and for his early feedback on the Battery Archive public site. We thank Prof. Venkat Subramanian of UT-Austin for supplying the model data used in Fig. 3. Sandia National Laboratories is a multi-mission laboratory managed and operated by The National Technology \& Engineering Solutions of Sandia, LLC, which is a wholly owned subsidiary of Honeywell International Inc., for the U.S. Department of Energy's National Nuclear Security Administration under contract DE-NA0003525. This paper describes objective technical results and analysis. Any subjective views or opinions that might be expressed in the paper do not necessarily represent the views of the U.S. Department of Energy or the United States Government. SAND2021-2617 O

\section{References}

[1] Energy Storage Grand Challenge Roadmap, 2020. https://www.energy.gov/sites/prod/files/2020/12/f81/Energy\%20Storage\%20Grand\%20Challenge $\% 20$ Roadmap.pdf (accessed February 1, 2021).

[2] Sulzer, V., Marquis, S. G., Timms, R., Robinson, M., and Chapman, S. J. (2020, February 7). Python Battery Mathematical Modelling (PyBaMM). ECSarXiv.

[3] CellPy Package. https://github.com/iepegit/cellpy (accessed February 1, 2021).

[4] Herring, P., Gopal, C.B., Aykol, M., Montoya, J.H., Anapolsky, A., Attia, P.M., Gent, W., Hummelshøj, J.S., Hung, L., Kwon, H.-K., Moore, P., Schweigert, D., Severson, K.A., Suram, S., Yang, Z., Braatz, R.D., and Storey, B.D., SoftwareX 2020, 11, 100506.

[5] Lewis-Douglas, A., Pitt, L., and Howey, D.A. (2020, October 28). Galvanalyser: A Battery Test Database. arXiv:2010.14959v1 [eess.SY]

[6] Universal Battery Database. https://github.com/Samuel-Buteau/universal-battery-database (accessed February 1, 2021)

[7] D3.js Library. https://d3js.org/

[8] Plotly Javascript Open Source Graphing Library. https://plotly.com/javascript/

[9] Cube.js. https://cube.dev/ 
[10] Redash. https://github.com/getredash/redash

[11] Redash - Setup \& Querying for JSON APIs. https://redash.io/help/data-sources/querying/json-api

[12] Postgres Table Updates. https://dba.stackexchange.com/questions/84802/slow-postgres-updates-ifother-columns-are-indexed (accessed February 1, 2021)

[13] PostgreSQL 9.4 - JSON Functions and Operators. https://www.postgresql.org/docs/9.4/functionsjson.html (accessed February 1, 2021)

[14] PostgreSQL 9.5 - TABLESAMPLE Implementation. https://wiki.postgresql.org/wiki/TABLESAMPLE Implementation (accessed February 1, 2021)

[15] CALCE Battery Research Group. https://web.calce.umd.edu/batteries/data.htm (Accessed February 1, 2021)

[16] He, W., Williard, N., Osterman, M., and Pecht, M. J. Power Sources 2011, 196, 10314.

[17] Devie, A., Baure, G., and Dubarry, M. Energies 2018, 11, 1031.

[18] Oxford Battery Degradation Dataset 1, 2017. https://doi.org/10.5287/bodleian:KO2kdmYGg

[19] Birkl, C.R. "Diagnosis and Prognosis of Degradation in Lithium-lon Batteries", PhD thesis, Department of Engineering Science, University of Oxford, 2017.

[20] Preger, Y.; Barkholtz, H. M.; Fresquez, A.; Campbell, D. L.; Juba, B. W.; Romàn-Kustas, J.; Ferreira, S. R.; and Chalamala, B. R. J. Electrochem. Soc. 2020, 167, 120532.

[21] Juarez-Robles, D., Jeevarajan, J.A., and Mukherjee, P. J. Electrochem. Soc. 2020, 167, 160510.

[22] Heenan, T.M.M., Jnawali, A., Kok, M.D.R., Tranter, T.G., Tan, C., Dimitrijevic, A., Jervis, R., Brett, D.J.L., and Shearing, P.R. J. Electrochem. Soc. 2020, 167, 140530.

[23] Gun, D., Perez, H., and Moura, S. (2015), Fast Charging Tests, Dryad, Dataset, https://doi.org/10.6078/D1MS3X

[24] Saha, B. and Goebel, K. (2007). "Battery Data Set", NASA Ames Prognostics Data Repository (http://ti.arc.nasa.gov/project/prognostic-data-repository), NASA Ames Research Center, Moffett Field, CA 